\title{
Beam steering by digital micro-mirror device for multi-beam and single-chip lidar
}

Joshua Rodriguez, Braden Smith, Eunmo Kang, Brandon Hellman, Guanghao Chen, et al.

Joshua Rodriguez, Braden Smith, Eunmo Kang, Brandon Hellman, Guanghao Chen, Adley Gin, Alonzo Espinoza, Yuzuru Takashima, "Beam steering by digital micro-mirror device for multi-beam and single-chip lidar," Proc. SPIE 10757, Optical Data Storage 2018: Industrial Optical Devices and Systems, 107570F (14 September 2018); doi: 10.1117/12.2323757

Event: SPIE Optical Engineering + Applications, 2018, San Diego, California, United States 


\title{
Beam steering by digital micro-mirror device for multi-beam and single-chip lidar
}

\author{
Joshua Rodriguez ${ }^{\mathrm{a}}$, Braden Smith ${ }^{\mathrm{a}, \mathrm{b}}$, Eunmo Kang ${ }^{\mathrm{a}}$, Brandon Hellman ${ }^{\mathrm{a}}$, Guanghao Chen ${ }^{\mathrm{a}, \mathrm{c}}$, \\ Adley Gin ${ }^{\mathrm{a}}$, Alonzo Espinoza ${ }^{\mathrm{a}}$, and Yuzuru Takashima ${ }^{\mathrm{a}}$ \\ ${ }^{a}$ University of Arizona, Takashima Group, College of Optical Science, 1630 E. University \\ Blvd., Tucson, AZ, USA, 85719

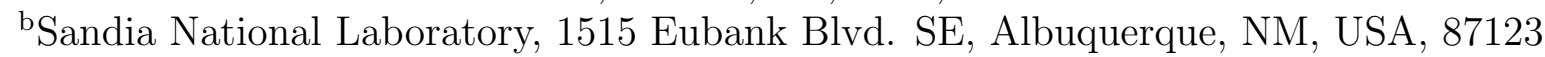 \\ ${ }^{\mathrm{c}}$ University of California San Diego, 9500 Gilman Dr, La Jolla, CA, USA, 92093
}

\begin{abstract}
A novel method of beam steering, utilizing a mass-produced Digital Micromirror Device (DMD), enables a reliable single chip Light Detection and Ranging (LIDAR) with a large field of view while having minimum moving components. In the single-chip LIDAR, a short-pulsed laser is fired in a synchronous manner to the micromirrors rotation during the transitional state. Since the pulse duration of the laser pulse is substantially short compared to the transitional time of the mirror rotation, virtually the mirror array is frozen in transition at several discrete points, which forms a programmable and blazed grating. The programmable blazed grating efficiently redirects the pulsed light to a single diffraction order among several while employing time of flight measurement. Previously, with a single $905 \mathrm{~nm}$ nanosecond laser diode and Si avalanche photo diode, a measurement accuracy and rate of $<1 \mathrm{~cm}$ and $3.34 \mathrm{k}$ points $/ \mathrm{sec}$, respectively, was demonstrated over a $1 \mathrm{~m}$ distance range with $48^{\circ}$ full field of view and 10 angular resolution. We have also increased the angular resolution by employing multiple laser diodes and a single DMD chip while maintaining a high measurement rate of $3.34 \mathrm{k}$ points $/ \mathrm{s}$. In addition, we present a pathway to achieve $0.65^{\circ}$ resolution with $60^{\circ}$ field of view and $23 \mathrm{k}$ points $/ \mathrm{s}$ measurement rate.
\end{abstract}

Keywords: optics, photonics, lasers, MEMS, DMD, LIDAR

\section{INTRODUCTION}

Laser beam steering technology is essential for Light Detection and Ranging (LIDAR) systems. For this reason, beam steering technologies have been actively researched. Along with mechanical and completely non-mechanical beam steering, Micro-Electro-Mechanical-Systems (MEMS) is an emerging beam steering field that is especially suitable for LIDAR systems. Mechanical scanning including gimbals, fast-steering mirrors, Risley prisms, rotating polygon mirrors and gratings have been used for wide wavelength ranges. ${ }^{1}$ Although mechanical beam scanning modalities are widely adopted, having fewer or no moving parts and smaller component inertia is more desirable for fast and compact beam steering devices so that size, weight, cost, and power consumption can be reduced. ${ }^{2,3}$ These qualities are especially required for autonomous vehicle and robotics applications. In contrast, completely non-mechanical scanning such as programmable spatial light modulators, modulo $2 \pi$ optical phased arrays, solid state phase arrays, and liquid crystal electro-optic scanners are emerging, and are now actively researched. ${ }^{2,4-7}$

In terms of reducing small component weight and inertia to fast beam steering, Micro-Electro-Mechanical Systems (MEMS) are promising due to their small size and weight, low production cost, high energy efficiency, and applicability to wide wavelength ranges. These MEMS devices include single resonant mirrors and shifting lenslet arrays. ${ }^{2,8,9}$ However, in LIDAR applications for autonomous vehicles, a large steering angle as well as large beam size are needed to cover a large angle of scanning and minimize beam divergence due to diffraction. Unfortunately, resonant mirrors and shifting lenslet arrays are limited in angular range and maximum accommodated beam size. Current high-end resonant mirror MEMS scanning systems have moderate fields of view at $36^{\circ}$ and scan rates of

Further author information: (Send correspondence to Joshua Rodriguez)

J.R.: E-mail: jrodriguez@optics.arizona.edu, Telephone: 19186067875

B.S.: E-mail: bradens@email.arizona.edu, Telephone: 15205895528

Optical Data Storage 2018: Industrial Optical Devices and Systems, edited by Ryuichi Katayama,

Yuzuru Takashima, Proc. of SPIE Vol. 10757, 107570F · (c) 2018 SPIE

CCC code: $0277-786 \mathrm{X} / 18 / \$ 18 \cdot$ doi: $10.1117 / 12.2323757$

Proc. of SPIE Vol. 10757 107570F-1 
$21 \mathrm{kHz} .^{2,10}$ However, a resonant mirror's maximum beam diameter is only increased at the expense of decreasing the maximum scan rate. ${ }^{9}$ An optical amplification of the steering angle by an inverse telescope design has been reported; however, this design requires a reduced beam diameter to conserve the Lagrange invariant, which would limit the effective delivery of light over large distances due to beam spreading by diffraction. ${ }^{11,12}$

As our group previously demonstrated, beam steering by Digital Micromirror Device (DMD) with a short pulsed laser is not only possible, but can be implemented in highly accurate scanning LIDAR systems. ${ }^{13}$ This beam steering technique has a high beam steering efficiency (close to $100 \%$ in theory), a larger beam size (same as DMD area), a wide field of view $\left(48^{\circ}\right)$, and a high scan rate (tens of $\mathrm{kHz}$ ) while minimizing the number of moving parts. The limitation of this approach, however, is that the maximum number of scanning points is limited to about 5 to 9 points for a wavelength range of 0.5 to $1 \mu \mathrm{m}$ with a commercially available DMD. We now propose and demonstrate increasing the angular resolution of the LIDAR system by implementing multiple laser diodes.

\section{MULTI-BEAM AND DISCRETE BEAM STEERING BY DMD}

\subsection{DMD Beam Steering Theory}

In Fig. 1(a), the DMD is schematically depicted. This beam steering setup utilizes a $608 \times 684$ (horizontal by vertical) DMD chip (DLP3000, Texas Instruments). The micromirrors are positioned in a diamond configuration with a corner-to-corner period of $10.8 \mu \mathrm{m}$ as shown in Fig. 1(a). On this DMD, an array of micromirrors flip between an "on" and "off" state, shown in Fig. 1(b)-(d), by rotating $\pm 12^{\circ}$ about an axis defined by the diagonal of the mirror. Thus, a DMD is designed for binary spatial light modulation and is not intended to be used for angular beam steering, unless additional optics to convert the spatial modulation to angular modulation are incorporated at the expense of light throughput. ${ }^{14}$

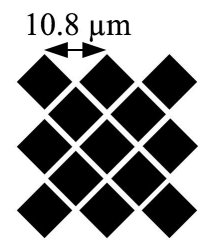

(a)

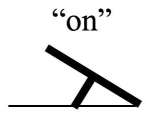

(b)

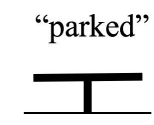

(c)

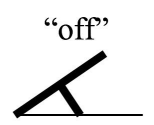

(d)

Figure 1. Representation of the (a) DMD diamond pixel layout (Top View); (b) a mirror in the "on" position at $+12^{\circ}$ (c) a mirror in the "parked" position at $0^{\circ}$ when the DMD is powered down; (d) a mirror in the "off" position at $-12^{\circ}$.

The unused transitional state of the DMD is utilized with a short-pulsed laser with a pulse duration much shorter than the transition time of the mirrors. For a particular steered beam angle, there is a corresponding delay between laser diode activation and DMD mirror flipping. The micromirror movement is frozen at an angle between the stationary on- and off-states with the pulsed laser. Thus, it is feasible to form a programmable and highly efficient blazed diffraction grating to discretely steer the laser beam.

\subsection{Multiple Beam Scanning}

Pulsed light from a 905nm and 8ns pulsed laser module (LS9-220-8-S10-00, Laser Components, Germany) is collimated by a $20 \mathrm{X}$ and 0.4 NA microscope objective lens, which illuminates DMD. The tilt timing of the DMD mirror is synchronously controlled with the laser pulse by an Arduino microcontroller with a DS1023 delay IC chip. By adjusting the time delay, each of the pulses are diffracted into one among 5 discrete diffraction orders with high diffraction efficiency, theoretically close to $100 \%$. The number of scanning points is increased by multiple laser sources as depicted in Fig. 2. Two additional laser modules are added with $\pm 3.3^{\circ}$ of incident angle deviated from the central laser path. This configuration allows qasi-evenly-spaced angular spacing. Figure 3 is a simulated intensity distribution over the $50^{\circ}$ field of view by Huygen-Fresnel integral. Each mirror was modeled as a series of point sources with an associated phase and optical path length (OPL) induced by the tilt of mirror while taking into account the angle of incidence of the laser module. 


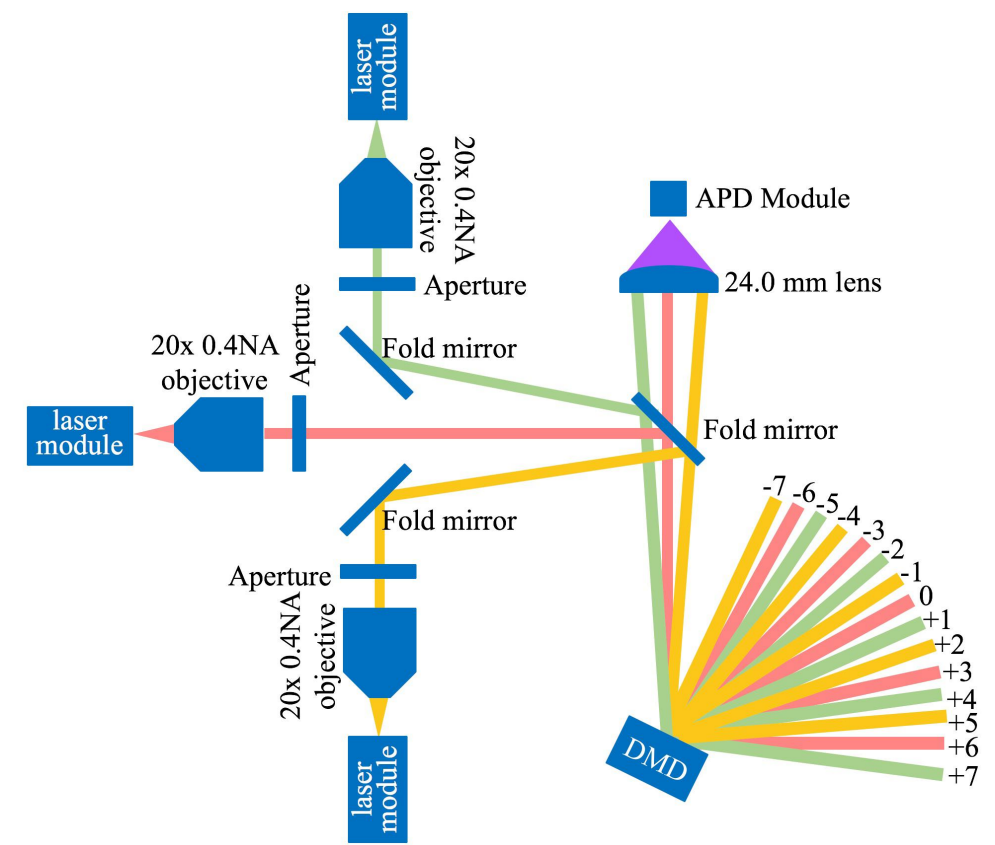

Figure 2. Schematic of setup utilizing 3 laser diodes.

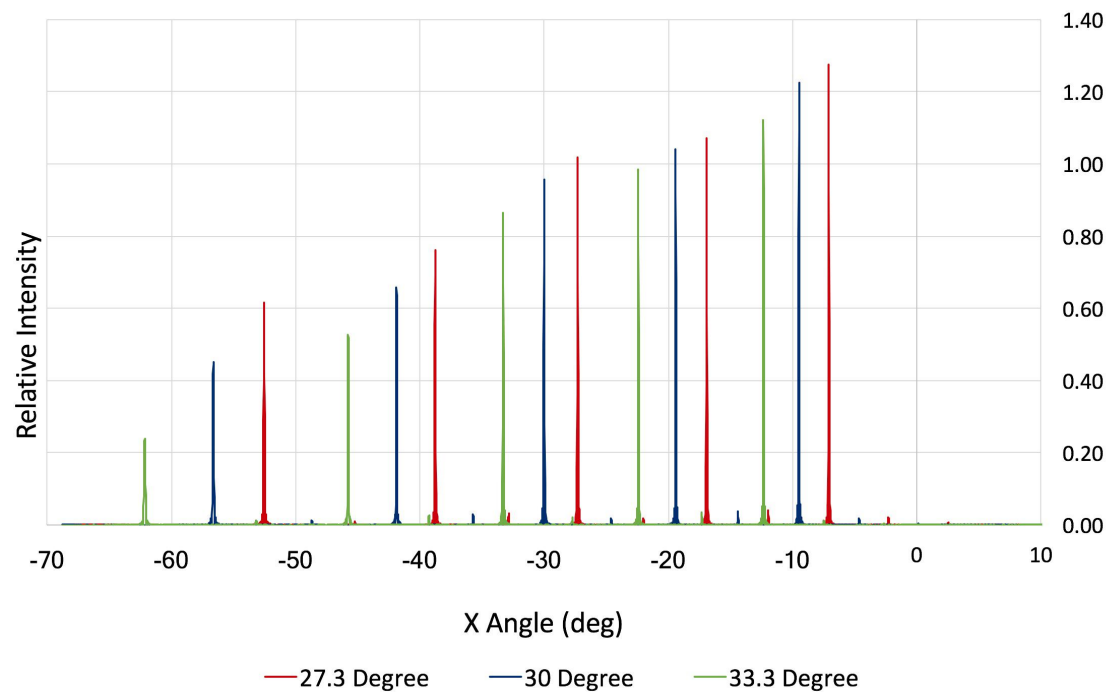

Figure 3. Simulated intensity profile at far-field from three laser diodes. Intensity is normalized to peak intensity of 0th order beam from the central laser module.

\section{RESULTS}

\subsection{Beam Steering}

The pulse timing from the 3 laser diodes modules is synchronized to transition of micro mirrors between on- and off-state by Arduino micro controller by the control sequence depicted in Figure 4. Figure 5 shows a picture of 15 beam spots. A screen was placed at a distance of $50 \mathrm{~cm}$ from the DMD, and the scanned spots are captured as a movie. Then, video frames corresponding to each of the scanning spots are cropped. At the bottom of the picture, a long exposure of all 15 scanning spots is depicted. 


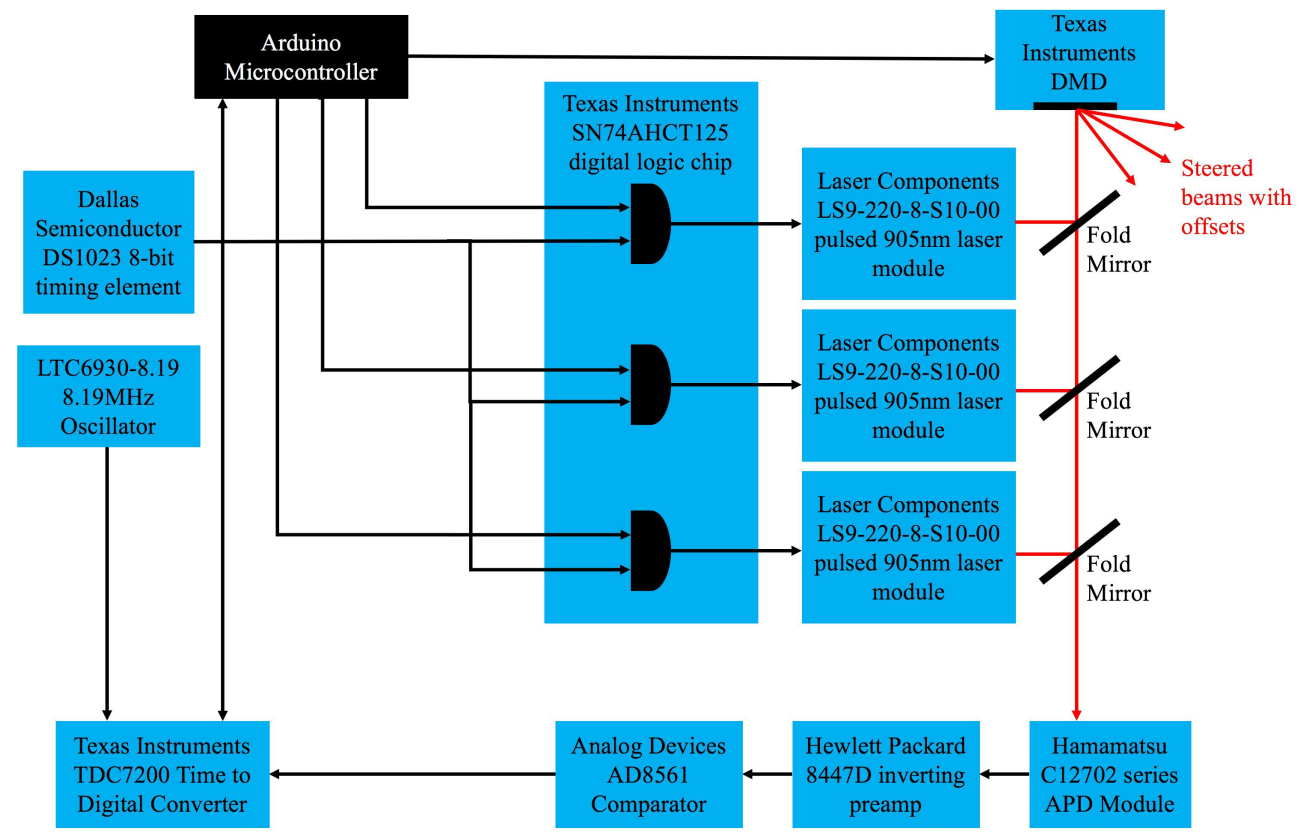

Figure 4. Block diagram of system operation with circuitry.

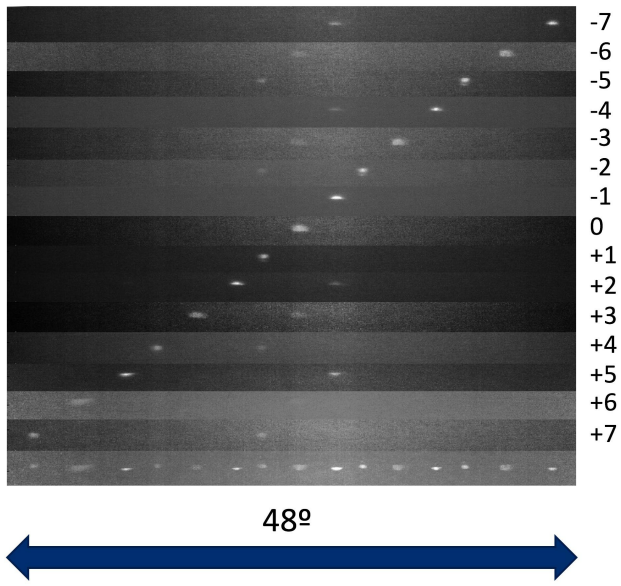

Figure 5. Total of 15 scanning spots from top to bottom, scanning point +7 to -7 depicted in Fig. 2(c) are captured. At the bottom of the picture, long exposure to show all the 15 scanning spots are depicted.

\subsection{Range Finding}

To make time of flight measurements for the LIDAR application, an avalanche photodiode (APD) (C12702, Hamamatsu) and a fold mirror were added to the single LD optical setup (similar to Fig. 2). In this experiment, multiple laser diodes were added to the system to demonstrate a pathway to increase the number of diffraction orders in such a system. The configuration shown in Fig. 2 involves three $905 \mathrm{~nm}$ laser diode module (LS9-2208-S10-00, Laser Components, Germany) directed towards the DMD with a $\pm 3.3^{\circ}$ incident angle separation from the central beam to ensure that the points are equally spaced. The $\pm 3.3^{\circ}$ incident angle separation generates equal spacing because diffraction orders are spaced approximately $10^{\circ}$ apart when using $905 \mathrm{~nm}$ light. To save space, fold mirrors were used to physically offset the three laser diodes while maintaining the correct angular spacing of the beams. 


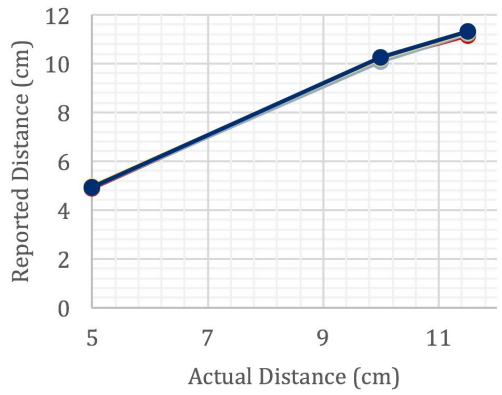

0
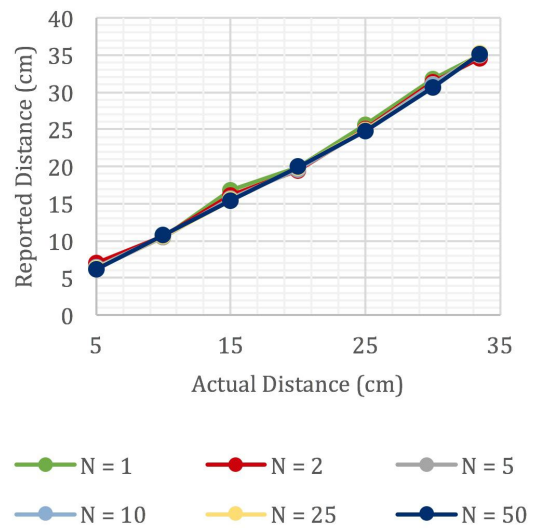

$-7$

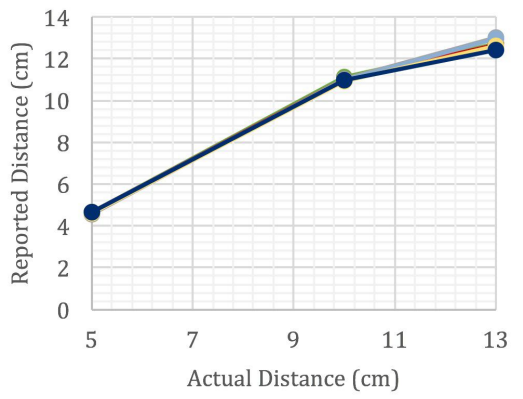

Figure 6. Distance measurements of objects placed in $+7,0$, and -7 diffraction orders. Measurement increments of $5 \mathrm{~cm}$ plus maximum distance measurable with system taken with averaging between $N=1$ and $N=50$ shown.

In Figure 6, distance measurement results from the central and outer orders are reported. As an object, we used pieces of cardboard aligned at each of the diffraction orders. The point denoted as +7 in Fig. 2 corresponds to the beam denoted as +7 in Figs. 6. Likewise, The point denoted as -7 in Fig. 2 corresponds to the beam denoted as -7 in Figs. 6 . The distance was measured at $5 \mathrm{~cm}$ increments in each case and additionally at the maximum measurable distance with the laboratory setup. As expected, the maximum measurable distance decreases in the outer orders since less power is redirected to them. The data accuracy stays fairly linear, with the accuracy staying well within $\pm 2 \mathrm{~cm}$ at all points with no averaging as shown in Fig. 6. With even some averaging, the data is more accurate.

\section{DISCUSSIONS}

The number of scan points is limited not only to a certain number of diffraction orders generated per laser diode, but also to the number of laser diodes used. With the current DMD (DLP3000), five diffraction orders are available when using a single $905 \mathrm{~nm}$ laser at a $30^{\circ}$ incidence angle. The number of scanning angles can be increased by employing a larger DMD micromirror pitch and/or a shorter wavelength. ${ }^{13}$ The limitations of two commercially available DMDs under ideal conditions for certain wavelengths of light are shown in Table 1. However, it is evident that we can accurately measure distance over $175 \mathrm{~m}$ with a higher scanning rate of up to $\left(256\right.$ lines/s) x 90 (points/line) $=23 \mathrm{k}$ points $/ \mathrm{sec}$ and a field of view over $60^{\circ}$ with $0.65^{\circ}$ angular resolution.

Table 1. Performance summary for two DMD types and two wavelengths of light. ${ }^{13}$

\begin{tabular}{ccccccccc}
\hline DMD Model & Wavelength & Range & $N_{L D}$ & $N_{\text {ord }}$ & $\begin{array}{c}\text { Notal } \\
\text { Scan Angles }\end{array}$ & FOV & Resolution & $\begin{array}{c}\text { Scan } \\
\text { Rate } \\
\text { (lines } / \mathrm{s})\end{array}$ \\
\hline DLP3000 & $905 \mathrm{~nm}$ & $55 \mathrm{~m}$ & 5 & 5 & 25 & $49^{\circ}$ & $1.9^{\circ}$ & 160 \\
DLP3000 & $1550 \mathrm{~nm}$ & $55 \mathrm{~m}$ & 8 & 3 & 24 & $50^{\circ}$ & $2.1^{\circ}$ & 167 \\
DLP9500 & $905 \mathrm{~nm}$ & $175 \mathrm{~m}$ & 11 & 7 & 77 & $48^{\circ}$ & $0.62^{\circ}$ & 299 \\
DLP9500 & $1550 \mathrm{~nm}$ & $175 \mathrm{~m}$ & 18 & 5 & 90 & $60^{\circ}$ & $0.65^{\circ}$ & 256
\end{tabular}

Currently, the angular spacing among multiple laser diodes is limited by the housing size of the laser diode modules and/or optics to collimate laser beam. Thus, it is not feasible to accommodate 18 lasers. Through an array of laser chips mounted on a common substrate with a single collimating lens solves the problem. The scanning speed is limited by the refresh rate of the DMD since a single pulse is launched per single rotation of the mirror. Thus, to scan 15 points, 15 DMD mirror flips is required. However, this requirement is not difficult. For an even faster scan rate, multiple pulses can be launched per single rotation of the DMD mirror that increase the scanning speed by a factor of the total number of diffraction orders. 


\section{CONCLUSIONS}

Multi-Beam Single-Chip DMD LIDAR provides a unique pathway to long distance range finding by leveraging commercially available Digital Micromirror Device. As a proof of concept, 15 points of range finding is demonstrated by using three $905 \mathrm{~nm}$ pulsed laser diode modules. With an array of laser diodes and state of the art detectors, we estimated that the system provides a solution for long range, high speed, and high angular resolution distance measurement without bulky scanning or receiving optics.

\section{REFERENCES}

[1] Stone, W. C., Stone, W. C., Juberts, M., Dagalakis, N., Stone, J., Gorman, J., Bond, P. J., Secretary, U., and Bement, A. L., "Performance analysis of next-generation ladar for manufacturing, construction, and mobility,"

[2] Cho, A. R., Han, A., Ju, S., Jeong, H., Park, J.-H., Kim, I., Bu, J.-U., and Ji, C.-H., "Electromagnetic biaxial microscanner with mechanical amplification at resonance," Opt. Express 23, 16792-16802 (Jun 2015).

[3] Niclass, C., Ito, K., Soga, M., Matsubara, H., Aoyagi, I., Kato, S., and Kagami, M., "Design and characterization of a 256x64-pixel single-photon imager in cmos for a mems-based laser scanning time-of-flight sensor," Opt. Express 20, 11863-11881 (May 2012).

[4] DeRose, C. T., Kekatpure, R. D., Trotter, D. C., Starbuck, A., Wendt, J. R., Yaacobi, A., Watts, M. R., Chettiar, U., Engheta, N., and Davids, P. S., "Electronically controlled optical beam-steering by an active phased array of metallic nanoantennas," Opt. Express 21, 5198-5208 (Feb 2013).

[5] Dou, R. and Giles, M., "Programmable phase grating and beam steerer by operating a lctv," in [Advanced Imaging Technologies and Commercial Applications], Clark, N. and Gonglewski, J., eds., 2566 (Aug. 1995).

[6] Davis, S., Rommel, S., Johnson, S., Farca, G., Rebolledo, N., Selwyn, S., and Anderson, M., "Electro-optic steering of a laser beam," SPIE Newsroom (2011).

[7] Ackerman, E., "Quanergy announces $\$ 250$ solid-state lidar for cars, robots, and more," IEEE Spectrum 7 (2016).

[8] Tuantranont, A., Bright, V., Zhang, J., Zhang, W., Neff, J., and Lee, Y., "Optical beam steering using memscontrollable microlens array," Sensors and Actuators A: Physical 91(3), 363 - 372 (2001). Proceedings of the Technical Digest of the 2000 Solid-State Sensors and Actuators Workshop.

[9] Inc., M. T., "Mirrorcle technologies mems mirrors-technical overview," Gimbal-les Two-Axis Scanning Micromirror Devices technical overview (2009).

[10] Sandner, T., Wildenhain, M., Gerwig, C., Schenk, H., Schwarzer, S., and Wölfelschneider, H., "Large aperture mems scanner module for 3d distance measurement," in [MOEMS and Miniaturized Systems IX], 7594, 75940D (Feb. 2010).

[11] Moss, R., Yuan, P., Bai, X., Quesada, E., Sudharsanan, R., Stann, B., Dammann, J., Giza, M., and Lawler, W., "Low-cost compact mems scanning ladar system for robotic applications," in [Laser Radar Technology and Applications XVII], 8379, 837903 (June 2012).

[12] Lee, X. and Wang, C., "Optical design for uniform scanning in mems-based 3d imaging lidar," Appl. Opt. 54, 2219-2223 (Mar 2015).

[13] Smith, B., Hellman, B., Gin, A., Espinoza, A., and Takashima, Y., "Single chip lidar with discrete beam steering by digital micromirror device," Opt. Express 25, 14732-14745 (Jun 2017).

[14] Instruments, T., "Dlp system optics," Application Report DLPA022 (2010). 